\title{
Risk factors for lymphedema and method of assessment in endometrial cancer: a prospective longitudinal multicenter study
}

Madelene Wedin, Karin Stalberg, Janusz Marcickiewicz, Eva Ahlner, Ulrika Ottander, Åsa Åkesson, Gabriel Lindahl, Ninnie Borendal Wodlin and Preben Kjølhede

The self-archived postprint version of this journal article is available at Linköping University Institutional Repository (DiVA):

http://urn.kb.se/resolve?urn=urn:nbn:se:liu:diva-180706

N.B.: When citing this work, cite the original publication.

Wedin, M., Stalberg, K., Marcickiewicz, J., Ahlner, E., Ottander, U., Åkesson, Å., Lindahl, G., Borendal Wodlin, N., Kjølhede, P., (2021), Risk factors for lymphedema and method of assessment in endometrial cancer: a prospective longitudinal multicenter study, International Journal of Gynecological Cancer, , ijgc-2021-002890. https://doi.org/10.1136/ijgc-2021-002890

Original publication available at:

https://doi.org/10.1136/ijgc-2021-002890

Copyright: BMJ Publishing Group

http://group.bmj.com/ 


\section{Risk factors for lymphedema and method of assessment in endometrial cancer. A prospective longitudinal multicenter study.}

authored by

Madelene Wedin 1, MD, (https://orcid.org/0000-0001-9940-4838), Karin Stålberg 2, MD, $\mathrm{PhD}$, (https://orcid.org/0000-0001-5527-8796), Janusz Marcickiewicz ${ }^{3}$, MD, PhD, (https://orcid.org/0000-0001-7512-185X), Eva Ahlner ${ }^{4}$, RPT, Ulrika Ottander ${ }^{5}, \mathrm{MD}, \mathrm{PhD}$, (https://orcid.org/0000-0002-1383-7753), Åsa Åkesson ${ }^{6}$, MD, (https://orcid.org/0000-00024938-2869), Gabriel Lindahl ${ }^{4}$, MD, PhD, (https://orcid.org/0000-0001-9494-1765), Ninnie Borendal Wodlin ${ }^{1}$, MD, PhD, (https://orcid.org/0000-0002-3730-7722), Preben Kjølhede ${ }^{1}$, $\mathrm{MD}, \mathrm{PhD}$ (https://orcid.org/0000-0001-5702-4116). On behalf of the LASEC study group

Affiliations:

${ }^{1}$ Department of Obstetrics and Gynecology in Linköping, and Department of Biomedical and Clinical Science. Linköping University, Linköping, Sweden

${ }^{2}$ Department of Women's and Children's Health. Uppsala University, Uppsala, Sweden

${ }^{3}$ Department of Obstetrics and Gynecology. Varberg Hospital, Varberg, Sweden

${ }^{4}$ Department of Oncology, and Department of Biomedical and Clinical Science. Linköping University, Linköping, Sweden

${ }^{5}$ Department of Clinical Sciences, Obstetrics and Gynecology, Umeå University, Umeå, Sweden.

${ }^{6}$ Department of Obstetrics and Gynecology, Sahlgrenska University Hospital, and Institute of clinical sciences, Sahlgrenska Academy at the University of Gothenburg, Gothenburg, Sweden

Corresponding author:

Madelene Wedin, MD

Department of Obstetrics and Gynecology

University hospital

58185 Linköping

Sweden

Phone +46101030000

E-mail address: Madelene.Wedin@regionostergotland.se 


\section{Abstract}

Objective. The aim of the study was to determine risk factors for lymphedema of the lower limbs, assessed by four methods, one year after surgery for endometrial cancer.

Methods. A prospective longitudinal multicenter study, conducted in 14 Swedish hospitals. Two-hundred-thirty-five women with endometrial cancer were included; 116 underwent surgery including lymphadenectomy and 119 had surgery without lymphadenectomy. Lymphedema was assessed preoperatively and one year postoperatively objectively by systematic circumferential measurements of the legs, enabling volume estimation addressed as 1) crude volume and 2) body mass index-standardized volume, or 3) clinical grading, and 4) subjectively by patient-reported perception of leg swelling. In volume estimation, lymphedema was defined as volume increase $\geq 10 \%$. Risk factors were analyzed using forward stepwise logistic regression models and presented as adjusted odds ratio (aOR) and 95\% confidence interval $(\mathrm{CI})$.

Results. Risk factors varied substantially, depending on the method of determining lymphedema. Lymphadenectomy was a risk factor for lymphedema when assessed by body mass index-standardized volume (aOR 14.4, 95\%CI 3.49-59.62), clinical grading (aOR 2.11, 95\%CI 1.04-4.29) and patient-perceived swelling (aOR 2.51, 95\%CI 1.33-4.73), but not when evaluated by crude volume. Adjuvant radiotherapy was only a risk factor for lymphedema when assessed by body mass index-standardized volume (aOR 15.02, 95\%CI 2.34-96.57). Aging was a risk factor for lymphedema when assessed by body mass index-standardized volume (aOR 1.07, 95\%CI 1.00-1.15) and patient-perceived swelling (aOR 1.06, 95\%CI (1.02-1.10), but not when assessed by crude volume or clinical grading. Increase in body mass index was a risk factor for lymphedema when estimated by crude volume (aOR 1.92, 95\%CI 1.36-2.71) and patient-perceived swelling (aOR 1.36, 95\%CI 1.11-1.66), but not by body mass index-standardized volume or clinical grading. 
The extent of lymphadenectomy was strongly predictive for development of lymphedema when assessed by body mass index-standardized volume and patient-perceived swelling, but not by crude volume or clinical grading.

Conclusion. Apparent risk factors for lymphedema differed considerably depending of the method to determine lymphedema. This highlights the need for a 'gold standard method when addressing lymphedema for determining of risk factors.

Key words: Endometrial cancer; Lymphedema; Risk factors, Surgery

\section{Highlights}

- $\quad$ Using different methods for assessing lymphedema disclosed different apparent risk factors.

- Leg volume should be body mass index-adjusted when used for assessing lymphedema.

- $\quad$ Aging, lymphadenectomy, and adjuvant radiation therapy were important risk factors for lymphedema. 


\section{Introduction}

Lymphedema of the lower limbs is a frequent long-term complication after treatment of gynecological malignancies $[1,2]$. The reported prevalence of lymphedema after treatment of endometrial cancer varies between $1 \%$ and $49 \%$ [3]. This considerable variation in prevalence is strongly associated with different definitions and methods of determining lymphedema [4,5]. Lymphadenectomy has been suggested as a major risk factor [6-8]. Although several other risk factors have been identified such as age [9,10], body mass index [6,11], extent of lymphadenectomy [12], adjuvant chemotherapy [5] and radiation therapy [7], the results are inconsistent. The discrepancies are mainly caused by the use of different definitions and methods of determining lymphedema. Several researchers have emphasized the need for a gold standard method for defining and determining lymphedema which would contribute to the estimation of true risk factors for lymphedema [2-4,13].

We have previously published the primary outcome of a prospective, longitudinal observational Swedish multicenter study, the Lymphedema After Surgery for Endometrial Cancer trial (the LASEC trial) that demonstrated variation in incidence of lymphedema, depending on the method of determining lymphedema [4]. The present study analyses a secondary objective of the LASEC trial.

The primary aim of this study was to determine risk factors for lymphedema one year after treatment of endometrial cancer, assessed by four different methods of assessing lymphedema; two by objectively using volume estimation of the legs, one by clinically grading of lymphedema performed by lymphedema therapist, and one subjectively by the patient-perception of leg swelling. 


\section{Methods}

The LASEC study, a prospective longitudinal observational multicenter study, was conducted between June 2014 and January 2018. Fourteen hospitals in Sweden participated; four university, six central, and four county hospitals. The Regional Ethics Board of Linköping University (Dnr 2013/373-31) approved the study and the study complies with the STROBE Statement requirements. The study was registered in ClinicalTrial.gov (ID: NCT02115477; initial release 04/13/2014).

Briefly, women diagnosed with presumed early stage endometrial cancer and assigned for primary surgery were eligible for the study. After oral and written consent were obtained, the participants underwent surgery and adjuvant oncological treatment according to the Swedish National Guidelines for Endometrial Cancer [14]. The surgery included pelvic and paraaortic lymphadenectomy in the women categorized as high-risk endometrial cancer whereas no lymph nodes were removed in the women categorized as low-risk endometrial cancer.

\section{Determination of lymphedema of the lower limbs}

All participants underwent assessments preoperatively (baseline) and one year after surgery including systematic measurement of the legs and clinical evaluation of lymphedema by a lymphedema therapist, and they filled in the Swedish version of the Lymphedema Quality of Life Questionnaire (LYMQOL) [15,16].

\section{Leg volume estimation}

Systematic measurement of leg circumference from a fixed point proximal to the malleolus to the groin with four cm intervals was conducted enabling calculation of estimated leg volume according to the cone model described by Sitzia et al [17]. The volume change from baseline to one year was classified as lymphedema if the increase was $\geq 10 \%$ in one of the legs and as no lymphedema if the increase was $<10 \%$ in both legs, according to the International Society 
of Lymphology definition [18]. Since body mass index per se is strongly correlated with leg volume [4] and the groups with and without lymphadenectomy in this study differed significantly in body mass index we standardized the estimated leg volumes on each occasion of assessment to the mean body mass index of the entire group of participants on the occasion (body mass index-standardized volume $\left.=\frac{\text { estimated leg volume } * \text { mean body mass index }}{\text { de facto body mass index }}\right)$ in order to outweigh the body mass index factor when determining the influence of surgery on development of lymphedema.

\section{Clinical grading of lymphedema}

Clinical grading of the legs was performed by lymphedema therapists according to the method described by Bruna et al [19]. Each leg was evaluated separately. The grading of lymphedema was carried out using a five-level scale ranging between Grade 0 and Grade 4. The change between the baseline and the one-year assessment was categorized as 'No lymphedema' provided both measurements in both legs were Grade 0 or as 'unchanged' if the grade determined as $>$ Grade 0 was similar on both occasions. The change was categorized as 'Lymphedema' if the one-year grading was higher than the baseline grading in one of the legs.

\section{Patient-perceived swelling of the legs}

The Swedish version of the LYMQOL for the legs contains a question concerning the perception of swelling of the legs [16]. The answer options to the question concerning swelling were restricted to four options: 'Not at all'; 'A little'; 'Quite a bit' or 'A lot'. The change in the degree of perceived swelling from baseline to the one-year assessment was classified as 'No perceived swelling' if both legs were graded 'Not at all' on both occasions, or as 'unchanged swelling' provided that the swelling was graded as 'A little' or more and similar on both occasions in both legs. The change was classified as 'Perceived swelling' provided that both legs at baseline were graded as 'Not at all' and at least one of the legs at 
the one-year assessment was graded as 'A little' or more, or if a swelling graded as at least 'A little' at baseline in one of the legs increased in grade of swelling at the one-year assessment.

\section{Data availability}

In accordance with the journal's guidelines, we will provide our data in deidentified form for the reproducibility of this study in other centers if such is requested.

\section{Statistics}

The statistical analyses were performed using the software TIBCO Statistica ${ }^{\mathrm{TM}}$, version 13.5 (TIBCO Software Inc, Palo Alto, CA 94303, USA). Continuous data are expressed as mean and one standard deviation (SD). Nominal data are described as number and frequency in percent.

Risk factors for lymphedema were divided into preoperative risk factors, intra - and postoperative risk factors, and lymphadenectomy-related risk factors. Initially the preoperative risk factors were evaluated individually in univariate logistic regression models and thereafter entered into a multiple forward stepwise logistic regression model. Secondly, those risk factors that turned out to be independent risk factors were further entered into a new multiple forward stepwise logistic regression model including the intra- and postoperative risk factors. Finally, in order to evaluate risk factors related to lymphadenectomy, each variable of lymphadenectomy was individually analyzed in a multiple forward stepwise logistic regression model adjusted for the independent risk factor revealed in the second step described above and factors that could potentially influence lymphadenectomy (body mass index, estimated intraoperative bleeding, American Society of Anesthesiologists classification, surgical method and mode of hysterectomy). The outcome of the logistic regressions is presented as crude or adjusted odds ratio (OR or aOR) and 95\% confidence interval (CI). 
The inter-rater agreement between the various methods of determining lymphedema was evaluated by means of the absolute agreement and Cohen's kappa. The kappa coefficient was interpreted according to Fleiss et al. [20] as: kappa coefficient values $\leq 0.40$ represent poor agreement beyond change; values $>0.40$ but $<0.75$ represent fair to good agreement beyond chance, and values $>0.75$ represent excellent agreement beyond chance.

\section{Statistical power}

The sample size of the LASEC trial was based on difference in incidence of lymphedema between the groups with and without lymphadenectomy. No specific estimation of the sample size was done in order to evaluate risk factors. 


\section{Results}

Two-hundred thirty-five women completed the one-year follow-up in the LASEC study of which 116 had undergone surgery with lymphadenectomy and 119 had had surgery without lymphadenectomy. An increase in body mass index-standardized volume of $\geq 10 \%$ was seen in $9.5 \%(22 / 231)$ one year after surgery. The corresponding figure for an increase of $\geq 10 \%$ in crude leg volume was $12.1 \%$ (28/231). By clinical grading evaluation of the legs, $17.5 \%$ (40/229) had developed lymphedema, and 29.6\% (68/230) by the patient-perceived perception of leg swelling.

Assessment by body mass index-standardized volume increase $\geq 10 \%$ :

Table 1 presents the data of women with and without lymphedema assessed by body mass index-standardized volume increase $\geq 10 \%$. Of the preoperative factors, age (aOR 1.06, 95\%CI 1.00-1.13) and diabetes mellitus (aOR 3.69, 95\%CI 1.32-10.33) were found to be independent risk factors for lymphedema. When including intraoperative and postoperative factors they remained independent risk factors, although weaker than two other very strong risk factors: adjuvant radiotherapy (aOR 15.02, 95\%CI 2.34-96.57) and lymphadenectomy (aOR 14.42, 95\%CI 3.49-59.62).

The risk factors for lymphedema as related to lymphadenectomy are depicted in Table 2. For each lymph node removed, the risk of lymphedema increased by $3 \%$ (aOR 1.03 ; 95\%CI 1.01-1.05). Compared with removal of $\leq 10$ lymph nodes, all categories of number of removed lymph nodes were significant risk factors with the category 11-25 lymph nodes as the strongest risk factor (aOR 18.60, 95\%CI 3.31-104.45). Compared with no lymphadenectomy, pelvic lymphadenectomy only was a stronger risk factor than pelvic combined with paraaortic lymphadenectomy (aOR 21.84; 95\%CI 3.93-121.39 versus aOR 12.07; 96\%CI 2.92-49.87). Paraaortic lymphadenectomy up to the level of the left renal vein in general increased the risk for lymphedema. However, this was mainly attributed to 
lymphadenectomy to the level below the inferior mesenteric artery (aOR 25.12, 95\%CI3.54178.15). To include lymphadenectomy distal to the deep circumflex iliac vein was a stronger risk factor than to conclude the lymphadenectomy proximal to the vein $(\mathrm{aOR} 15.89,95 \% \mathrm{CI}$ 1.80-139.99 versus aOR 14.26, 95\%CI 3.41-59.69).

Assessment by crude volume increase $\geq 10 \%$ :

The data of women with and without lymphedema assessed by crude volume increase $\geq 10 \%$ are reported in Table 1. Medication with diuretics was found to be an independent preoperative risk factors for lymphedema (aOR 2.67, 95\%CI 1.04-6.89) whereas obesity was protective for developing lymphedema (aOR 0.88, 95\%CI 0.80-0.97). Both remained independent risk factors in the second statistical model where the intra- and postoperative factors were added (aOR 3.73, 95\%CI 1.33-10.43 and aOR 0.15, 95\%CI 0.04-0.53, receptively). Together with the increase (one year postoperatively - baseline) in body mass index (aOR 1.92, 95\% CI 1-36-2.71), the effect of these factors eliminated the effect of lymphadenectomy on development of lymphedema in the model. Thus, lymphadenectomy was not found to be a risk factor for lymphedema determined by $\geq 10 \%$ crude volume increase. Nor were any of the factors associated with lymphadenectomy found to be risk factors for lymphedema (Table 2).

\section{Assessment by clinical grading}

Table 3 demonstrates the data of women with and without lymphedema as determined by clinical grading. The analysis revealed no preoperative risk factors. Only lymphadenectomy was found to be an intra- and postoperative risk factor (aOR 2.11, 95\%CI 1.04-4.29). Of the factors associated with lymphadenectomy only the level of paraaortic lymphadenectomy was found to be a risk factor, particularly when the lymphadenectomy was carried out to the level of the inferior mesenteric artery (aOR 11.43, 95\%CI 3.28-39.87) (Table 4). Assessment by patient-perceived swelling of the legs 
The data on risk factors for patient-perceived swelling are presented in Table 3. Only age proved to be an independent risk factor of the preoperative factors (aOR 1.05; 95\% CI 1.011.08). When adding the intra- and postoperative factors to the model, age was still an independent risk factor (aOR 1.06, 95\%CI 1.02-1.10) along with lymphadenectomy (aOR $2.51,95 \%$ CI 1.34-4.73). An increase in body mass index from baseline to one year postoperatively, as well as severe postoperative complications (Clavien-Dindo $\geq$ grade 3 ) were independent risk factors (aOR 1.36, 95\%CI 1.11-1.66 and aOR 6.04, 95\%CI 1.57-23.22, respectively). As shown in Table 4, all factors associated with lymphadenectomy proved to be independent risk factors for lymphedema.

\section{Interrater agreement between methods of measuring lymphedema}

The absolute agreements between the various methods of determining lymphedema were high between $70 \%$ and $91 \%$ (Table 5), but by taking into account the by chance contribution most of the Cohen's kappa coefficients were low $(\leq 0.40)$ indicating poor agreement. Only body mass index-standardized volume and crude volume showed a fair to good agreement beyond chance $($ kappa $=0.55)$. 


\section{Discussion}

Summary of Main Results

We found a substantial variation in estimation of risk factors of lymphedema depending on the method. While lymphadenectomy and adjuvant radiotherapy were very strong independent risk factors for lymphedema when determining lymphedema after body mass index-standardized volume increase, lymphadenectomy was still an independent risk factor, although weaker, when determining lymphedema after clinical grading and patient-perceived swelling, but adjuvant radiotherapy was no longer an independent risk factor in these two modalities. Neither lymphadenectomy nor adjuvant radiotherapy were independent risk factors for lymphedema when measured as crude volume increase. On the other hand, obesity was a protective factor, and increase in body mass index and use of diuretics were much stronger risk factors that eliminated the effect of lymphadenectomy as a risk factor for lymphedema. This eliminating effect was also seen when determining risk factors associated with lymphadenectomy, where none of the lymphadenectomy factors were found to be predictive for lymphedema. The opposite was found when addressing lymphedema by means of body mass index-standardized and patient-perceived swelling. The number of dissected lymph nodes and the extent of the lymphadenectomy were all consistently strong predictive factors for lymphedema. The results in this respect concerning lymphedema as measured by clinical grading were inconsistent and consequently difficult to interpret clinically.

\section{Results in the Context of Published Literature}

To the best of our knowledge, this is the first study that evaluates risk factors for lymphedema based on four methods of measurements of lymphedema. Several studies have evaluated risk factors for lymphedema after treatment of endometrial cancer but the prerequisites vary substantially in methods and follow-up periods [5-8,10-12]. None of the risk factors found in the present study occurred simultaneously in all four models. Consistent with most of the other studies we found that lymphadenectomy was a risk factor when lymphedema was 
assessed by body mass index-standardized volume, clinical grading and patient-perceived swelling, and adjuvant radiotherapy was a strong treatment-related risk factor for lymphedema, when assessed by body mass index-standardized volume. Another important observation in this study was the association between increasing age and lymphedema determined by body mass index-standardized volume and patient-perceived swelling. The association between age and lymphedema is contradictory in the literature $[1,5,6,9,10,12,21$ 23], but aging causes substantial changes in the lymphatic system and consequently might contribute to development of lymphedema [24]. Diabetes was a risk factor for lymphedema when assessed by body mass index-standardized volume. The association between diabetes and lymphedema is well known, but the pathophysiological mechanisms are not yet completely elucidated [25].

The reports on body mass index as a risk factor for lymphedema are contradictory $[1,5.6,11,12,21-23]$. While neither preoperative body mass index nor body mass index increase were risk factors for lymphedema when determined by body mass indexstandardized volume or clinical grading, the body mass index increase was a risk factor in patient-perceived swelling and clinical grading, and obesity was a protective factor for lymphedema determined by crude volume increase. Preoperative body mass index and increase in body mass index seemed to outweigh the effect of lymphadenectomy in the risk factor estimation using crude volume. Since body mass index is strongly positively correlated with leg volume [26] the findings concerning obesity and increase in body mass index may be caused by the selected definition of lymphedema as $\geq 10 \%$ volume increase. If an increase in leg volume in a normal weight woman reaches, for instance $400 \mathrm{~mL}$ to be defined as lymphedema, it is obvious that the same volume increase in an obese will not be detected as lymphedema. The incidence of lymphedema may therefore be underestimated when determined by crude volume increase, and consequently the risk factors may be misleading. 
These arguments underline the importance of using body mass index-standardizing leg volumes in order to describe lymphedema as determined as $\geq 10 \%$ volume increase.

The number of removed lymph nodes constituted a risk factor, with an increase in odds ratio of $3 \%$ per lymph node removed. This is consistent with other reports [1,5,12,22,23]. A systematic review and meta-analysis have suggested a lower risk for lymphedema after sentinel lymph node (SLN) technique in endometrial cancer [27]. However, the vast majority of the studies included in the review were retrospective studies and none were randomized controlled trials. The SLN technique was not applied in the present study. Whether the effect on the development of lymphedema of extirpation of specific and selected lymph nodes in the lymphatic pathway, such as SLNs, is comparable to that of the resection of a few unselected lymph nodes in the lymphatic pathway remains to be determined.

\section{Strengths and Weaknesses}

There are strengths as well as limitation of this study. The main strength besides the multicenter, prospective longitudinal design is the consistently used methodology for measuring lymphedema and including women with and without lymphadenectomy. Limitations might be selection bias and the sample size, as the sample size of the study was originally dimensioned to assess incidence and not risk factors for lymphedema. Another limitation is the follow-up period of one year as lymphedema may develop and progress later.

\section{Implications for Practical and Future Research}

Information on risk factors for lymphedema, and how to prevent and treat lymphedema is important in the preoperative counselling. Due to the inconsistent findings of incidences of lymphedema, risk factors and correlations between the incidences of lymphedema and the different measurement methods it seems inappropriate to use crude volume increase, clinical grading and patient's perception of leg swelling for estimation of the risk factors of lymphedema in clinical practice. In the light of the robust clinical risk factors that were 
established by using body mass index-standardized volume for determining lymphedema, we suggest that this method should be considered as the standard method for risk factor determination of lymphedema of the lower extremities.

\section{Conclusion}

Lymphadenectomy and adjuvant radiotherapy are strong treatment-related risk factors for lymphedema. Estimation of risk factors for lymphedema after treatment of endometrial cancer indicated a substantial association with measurement methods of lymphedema. This strongly raises questions on the clinical usefulness of risk factors if they are not consistent and highlights the needs for a 'gold standard` method when addressing lymphedema. 


\section{Contribution to authorship}

PK conceptualized the study and wrote the study protocol together with EA, KS, and JM. MW is the main author of the manuscript. PK and MW conducted the statistical analyses. All authors participated actively in conducting the study, evaluating the analyses and critically revised the manuscript, approved the final version, and are accountable for all aspects of the work.

\section{Conflict of interest statement}

The authors declare that there is no conflict of interest with regard to the publication of this article. 


\section{References}

1. Hareyama H, Hada K, Goto K, et al. Prevalence, classification, and risk factors for postoperative lower extremity lymphoedema in women with gynecologic malignancies: a retrospective study. Int J Gynecol Cancer. 2015;25(4):751-7. doi: 10.1097/IGC.0000000000000405.

2. Dessources K, Aviki E, Leitao MM Jr. Lower extremity lymphoedema in patients with gynecologic malignancies. Int J Gynecol Cancer. 2020;30(2):252-60. doi: 10.1136/ijgc2019-001032.

3. Lindqvist E, Wedin M, Fredrikson M, et al. Lymphoedema after treatment for endometrial cancer - A review of prevalence and risk factors. Eur J Obstet Gynecol Reprod Biol. 2017;211:112-21. doi: 10.1016/j.ejogrb.2017.02.021.

4. Wedin M, Stålberg K, Marcickiewicz J, et al. Incidence of lymphedema in the lower limbs and lymphocyst formation within one year of surgery for endometrial cancer: A prospective longitudinal multicenter study. Gynecol Oncol. 2020;159(1):201-8. doi: 10.1016/j.ygyno.2020.07.014.

5. Pigott A, Obermair A, Janda M, et al. Incidence and risk factors for lower limb lymphedema associated with endometrial cancer: Results from a prospective, longitudinal cohort study. Gynecol Oncol. 2020;158(2):375-81. doi: 10.1016/j.ygyno.2020.04.702

6. Yost KJ, Cheville AL, Al-Hilli MM, et al. Lymphedema after surgery for endometrial cancer: prevalence, risk factors, and quality of life. Obstet Gynecol. 2014;124(2 Pt 1):307-15. doi: 10.1097/AOG.0000000000000372.

7. Todo Y, Yamazaki H, Takeshita S, et al. Close relationship between removal of circumflex iliac nodes to distal external iliac nodes and postoperative lower-extremity 
lymphedema in uterine corpus malignant tumors. Gynecol Oncol. 2015;139(1):160-4. doi: 10.1016/j.ygyno.2015.07.003.

8. Mitra D, Catalano PJ, Cimbak N, et al. The risk of lymphedema after postoperative radiation therapy in endometrial cancer. J Gynecol Oncol. 2016;27(1):e4. doi: 10.3802/jgo.2016.27.e4.

9. Deura I, Shimada M, Hirashita K, et al. Incidence and risk factors for lower limb lymphedema after gynecologic cancer surgery with initiation of periodic complex decongestive physiotherapy. Int J Clin Oncol. 2015;20(3):556-60. doi: 10.1007/s10147014-0724-0.

10. Yoshihara M, Shimono R, Tsuru S, et al. Risk factors for late-onset lower limb lymphedema after gynecological cancer treatment: A multi-institutional retrospective study. Eur J Surg Oncol. 2020;46(7):1334-8. doi: 10.1016/j.ejso.2020.01.033.

11. Mendivil AA, Rettenmaier MA, Abaid LN, et al. Lower-extremity lymphedema following management for endometrial and cervical cancer. Surg Oncol. 2016;25(3):200-4. doi: 10.1016/j.suronc.2016.05.015.

12. Beesley VL, Rowlands IJ, Hayes SC, et al; Australian National Endometrial Cancer Study Group. Incidence, risk factors and estimates of a woman's risk of developing secondary lower limb lymphedema and lymphedema-specific supportive care needs in women treated for endometrial cancer. Gynecol Oncol. 2015;136(1):87-93. doi: 10.1016/j.ygyno.2014.11.006.

13. Biglia N, Zanfagnin V, Daniele A, et al. Lower Body Lymphedema in Patients with Gynecologic Cancer. Anticancer Res. 2017;37(8):4005-15. doi: 10.21873/anticanres.11785.

14. Swedish National guidelines for endometrial cancer (Nationellt vårdprogram livmodercancer) ([In Swedish]: 
https://www.cancercentrum.se/globalassets/cancerdiagnoser/gynekologi/livmoderkropp $\underline{\text { scancer/vardprogram/nationellt-vardprogram-endometriecancer.pdf }}$ (accessed April 26; 2021).

15. Keeley V, Crooks S, Locke J, et al. A quality of life measure for limb lymphoedema (LYMQOL). J Lymphoedema. 2010;5(1):26-37.

16. Wedin M, Fredrikson M, Ahlner E, et al. Validation of the Lymphoedema Quality of Life Questionnaire (LYMQOL) in Swedish cancer patients. Acta Oncol. 2020;59(3):365-71. doi: 10.1080/0284186X.2019.1701199.

17. Sitzia J. Volume measurement in lymphoedema treatment: examination of formulae. Eur J Cancer Care (Engl). 1995;4(1):11-6. doi: 10.1111/j.1365-2354.1995.tb00047.x

18. Executive Committee of the International Society of Lymphology. The diagnosis and treatment of peripheral lymphedema: 2020 Consensus Document of the International Society of Lymphology. Lymphology. 2020;53 1(3):3-19

19. Bruna J, Miller AJ, Beninson J. A simple clinical classification of lymphoedema. Eur J Plast Surg .1999);22:404-5.

20. Fleiss JL, Levin B, Cho Paik M. The measurement of interrater agreement. In: Statistical methods for rates and proportions. Eds, Fleiss JL, Levin B, Cho Paik M. 3rd Edition. John Wiley \& Sons Inc. Hoboken, New Jersey, USA. ISBN: 978-0-471-526292. October 2003. Page 604.

21. Kuroda K, Yamamoto Y, Yangisawa M et al. Risk factors and a prediction model for lower limb lymphedema following lymphadenectomy in gynecologic cancer: a hospitalbased retrospective cohort study. BMC Women's Health. 2017;17:50. doi:

10.1186/s12905-017-0403-1. 
22. Kunitake T, Kakuma T, Ushijima. Risk factors for lower limb lymphedema in gynecologic patients after initial treatment. Int J Clin Oncol. 2020;25(5):963-71. doi: 10.1007/s10147-019-01608-6.

23. Carlson LW, Kauderer J, Hutson A et al. GOG-244-The lymphedema and gynecological cancer (leg) study: Incidence and risk factors in newly diagnosed patients. Gynecol Oncol. 2020:156(2):467-74. doi: 10.1016/j.ygyno.2019.10.009.

24. Guliyeva G, Huayllani MT, Boczar D, et al. Age as risk factor for breast cancer-related lymphedema: a systematic review. J Cancer Surviv. 2021; Jan 24. doi: 10.1007/s11764021-00994-z.Online ahead of print.

25. McIntosh C, Green T. An overview of lower limb lymphoedema and diabetes. $J$ Lymphoedema. 2009;4(1):49-58.

26. Podleska LE, Poeppel T, Herbrik M, et al. Drug dosage in isolated limb perfusion: evaluation of a limb volume model for extremity volume calculation. World J Surg Oncol. 2014;12:81. doi: 10.1186/1477-7819-12-81.

27. Helgers RJA, Winkens B, Slangen BFM, et al. Lymphedema and post-operative complications after sentinel lymph node biopsy versus lymphadenectomy in endometrial carcinomas-A systematic review and meta-analysis. J Clin Med. 2020;10(1):120. doi: 10.3390/jcm10010120. 
Table 1. Risk factors for lower limb lymphedema determined as a volume increase $\geq 10 \%$ based on body mass index-standardized leg volume or

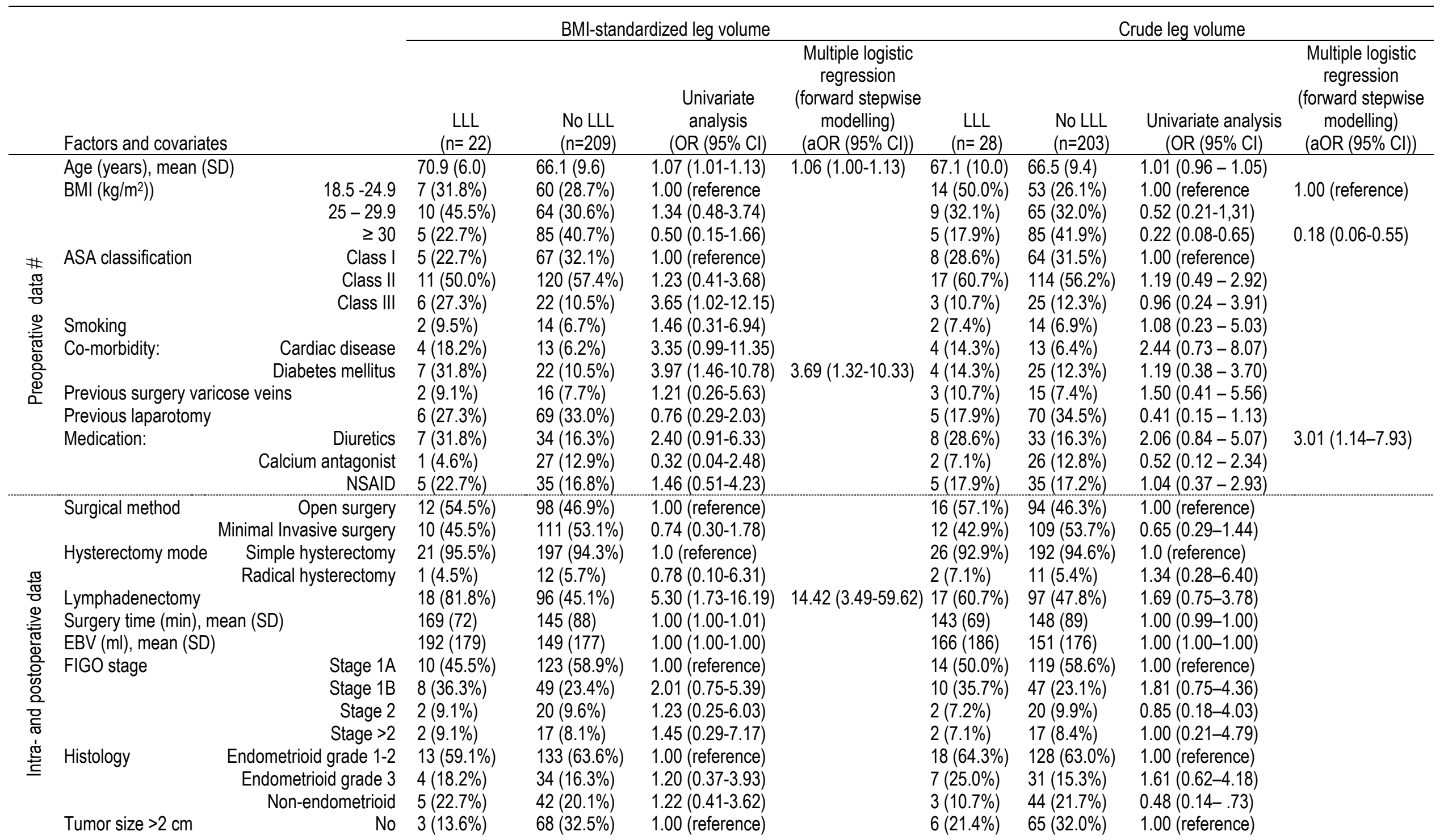




\begin{tabular}{|c|c|c|c|c|c|c|c|c|c|}
\hline & Yes & $17(77.3 \%$ & $102(48.8 \%)$ & $3.78(1.07-13.39)$ & & $17(60.7 \%)$ & $102(50.3 \%)$ & $1.81(0.68-4.82)$ & \\
\hline No informa & n available & $2(9.1 \%)$ & $39(18.7 \%)$ & $1.16(0.19-7.26)$ & & $5(17.9 \%)$ & $36(17.7 \%)$ & $1.50(0.43-5.28)$ & \\
\hline Myometrial invasion >50\% & & $11(50.0 \%)$ & $68(32.5 \%)$ & $2.07(0.86-5.02)$ & & $12(42.9 \%)$ & $67(33.0 \%)$ & $1.52(0.68-3.40)$ & \\
\hline Lymph node metastasis & & $1(4.5 \%)$ & $14(6.7 \%)$ & $0.66(0.08-5.30)$ & & $1(3.6 \%)$ & $14(6.9 \%)$ & $0.50(0.06-3.96)$ & \\
\hline \multirow[t]{4}{*}{ Clavien-Dindo classification* } & Grade 0 & $15(68.2 \%)$ & $153(73.2 \%)$ & 1.00 (reference) & & $23(82.1 \%)$ & $145(71.4 \%)$ & 1.00 (reference) & \\
\hline & Grade 1 & $3(13.6 \%)$ & $28(13.4 \%)$ & $1.09(0.30-4.02)$ & & $2(7.2 \%)$ & $29(14.3 \%)$ & $0.43(0.10-1.95)$ & \\
\hline & Grade 2 & $2(9.1 \%)$ & $19(9.1 \%)$ & $1.07(0.23-5.06)$ & & $2(7.1 \%)$ & $19(9.4 \%)$ & $0.66(0.14-3.04)$ & \\
\hline & $\geq$ Grade 3 & $2(9.1 \%)$ & $9(4.3 \%)$ & $2.27(0.45-11.47)$ & & $1(3.6 \%)$ & $10(4.9 \%)$ & $0.63(0.08-5.16)$ & \\
\hline \multirow[t]{3}{*}{ Adjuvant oncological treatment } & No & $12(54.6 \%)$ & $135(64.6 \%)$ & $2.27(0.45-11.47)$ & 1.00 (reference) & $1(3.6 \%)$ & $10(4.9 \%)$ & 1.00 (reference) & \\
\hline & Radiation & $3(13.6 \%)$ & $6(2.9 \%)$ & 1.00 (reference) & $15.02(2.34-96.57)$ & $19(67.9 \%)$ & $128(63.1 \%)$ & $3.37(0.78-14.61)$ & \\
\hline & motherapy & $5(22.7 \%)$ & $46(22.0 \%)$ & $5.62(1.25-25.37)$ & & $3(10.7 \%)$ & $6(3.0 \%)$ & $0.42(0.12-1.49)$ & \\
\hline \multirow{2}{*}{\multicolumn{2}{|c|}{ Chemotherapy + radiation }} & $2(9.1 \%)$ & $22(10.5 \%)$ & $1.22(0.41-3.66)$ & & $3(10.7 \%)$ & $4823.6 \%)$ & $0.96(0.26-3.54)$ & \\
\hline & & $-0.04(1.77)$ & $0.10(1.86)$ & $0.96(0.76-1.21)$ & & $1.40(1.60)$ & $-0.10(1.80)$ & $1.76(1.29-2.39)$ & $1.92(1.36-2.71)$ \\
\hline $\mathrm{BMI}\left(\mathrm{kg} / \mathrm{m}^{2}\right)$ & $\begin{array}{l}18.5-24.9 \\
25-29.9\end{array}$ & & & & & $\begin{array}{l}14(50.0 \%) \\
9(321 \%)\end{array}$ & $53(26.1 \%)$ & 1.00 (reference & 1.00 (reference) \\
\hline & $\geq 30$ & & & & & $5(17.9 \%)$ & $85(41.9 \%)$ & $0.22(0.08-0.65)$ & $0.15(0.04-0.53)$ \\
\hline Age (years), mean (SD) & & $70.9(6.0)$ & $66.1(9.6)$ & $1.07(1.01-1.13)$ & $1.07(1.00-1.15)$ & & & & \\
\hline Diabetes mellitus\# & & $7(31.8 \%)$ & $22(10.5 \%)$ & $3.97(1.46-10.78)$ & $5.44(1.67-17.66)$ & & & & \\
\hline Medication & Diuretics & & & & & $8(28.6 \%)$ & $33(16.3 \%)$ & $2.06(0.84-5.07)$ & $3.73(1.33-10.43)$ \\
\hline
\end{tabular}

a - adjusted. ASA - American Society of Anesthesiologist. BMI - body mass index. CI - confidence interval. EBV - estimated bleeding

volume. LLL - lower limb lymphedema. NSAID - non-steroidal anti-inflammatory drugs. OR - odds ratio. SD - standard deviation

* Clavien-Dindo classification of postoperative complications within 30 days.

$\S \Delta \mathrm{BMI}=\mathrm{BMI}$ at one year $-\mathrm{BMI}$ at baseline.

\# The preoperative data were analyzed separately in the first step. In addition to all factors and covariates depicted in the section intra- and postoperative data, the independent risk factors denoted under the section preoperative data were included in the multivariable analysis of intraand postoperative risk factors. Only aOR's of significant independent risk factors are denoted. 
Table 2. Risk factors for lymphedema related to lymphadenectomy. Lymphedema determined as a volume increase $\geq 10 \%$ based on body mass index-standardized leg volume or crude leg volume.

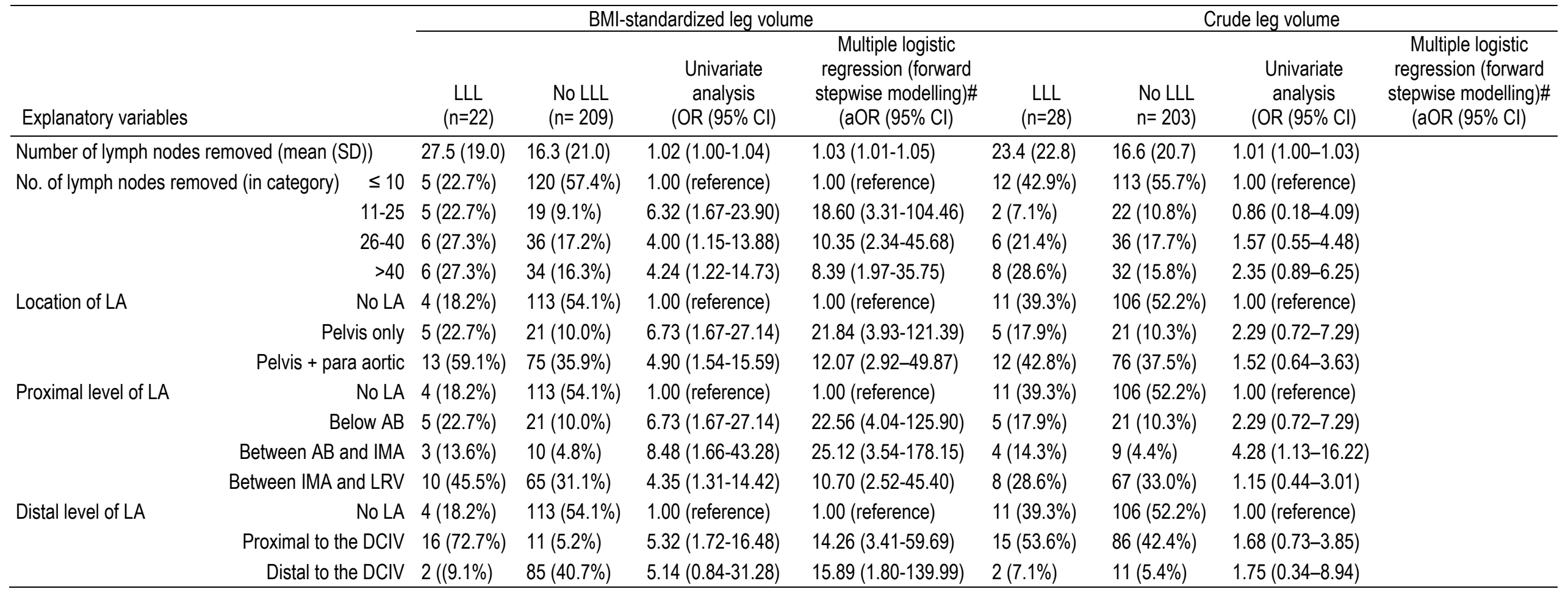

$\mathrm{a}$ - adjusted. AB - aortic bifurcation. CI - confidence interval. DCIV - deep circumflex iliac vein. LA - lymphadenectomy. LLL - lower limb lymphedema. LRV - left renal vein. IMA- inferior mesenteric artery. OR -odds ratio.

\#The multiple logistic regression forward stepwise models included adjustment for the independent risk factors depicted under intra- and postoperative data in Table 1 except the factor lymphadenectomy. Besides, potential factors that could influence lymphadenectomy (BMI, estimated bleeding volume, ASA classification, surgical method and mode of hysterectomy) were added simultaneously.

The explanatory variables depicted in Table 2 were analyzed individually in the multivariable models since these variabels may be intercorrelated. Only aORs of significant independent risk factors are denoted. 
Table 3. Risk factors for lower limb lymphedema determined by clinical grading or as patient's perception of increased swelling of the legs.

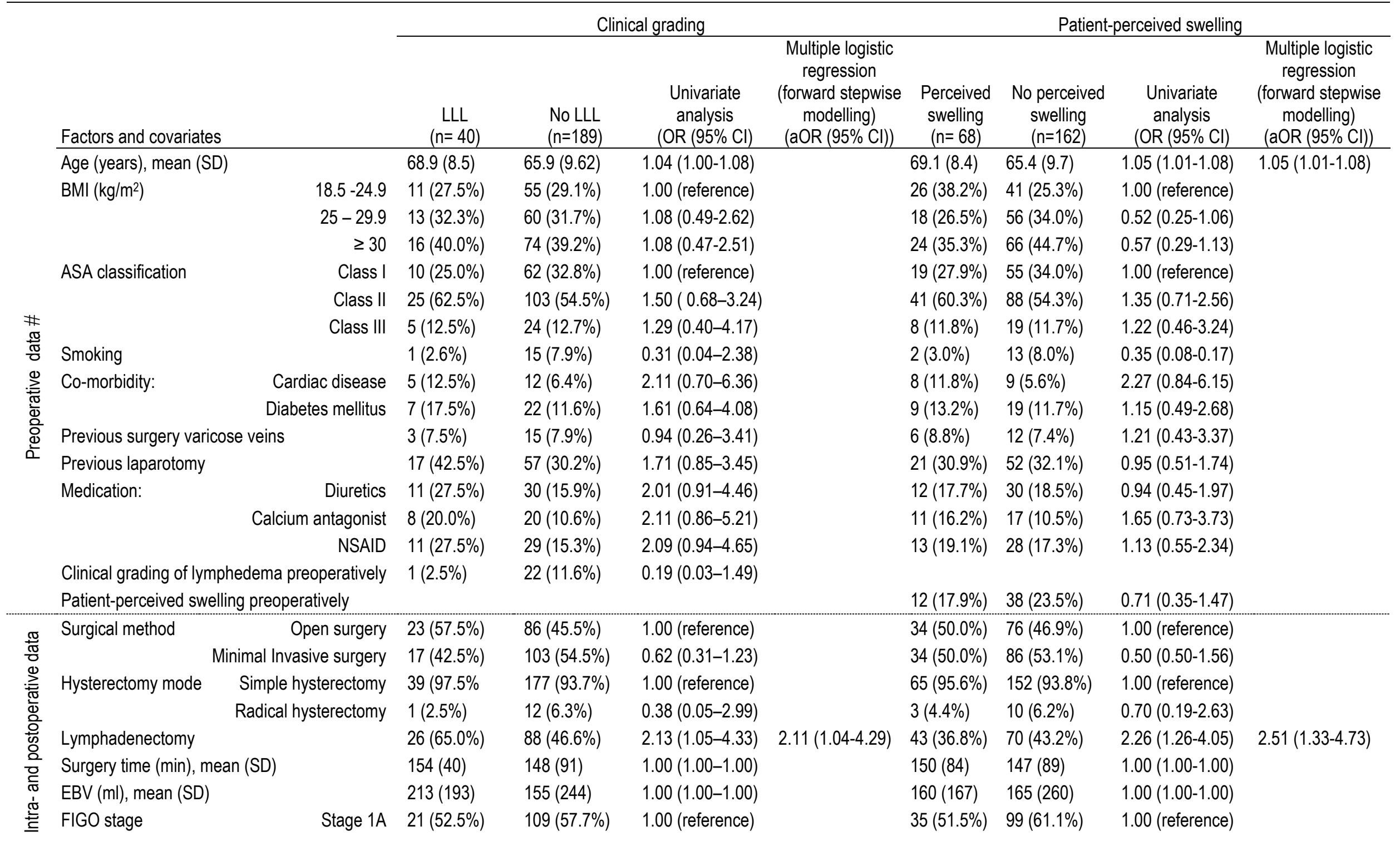




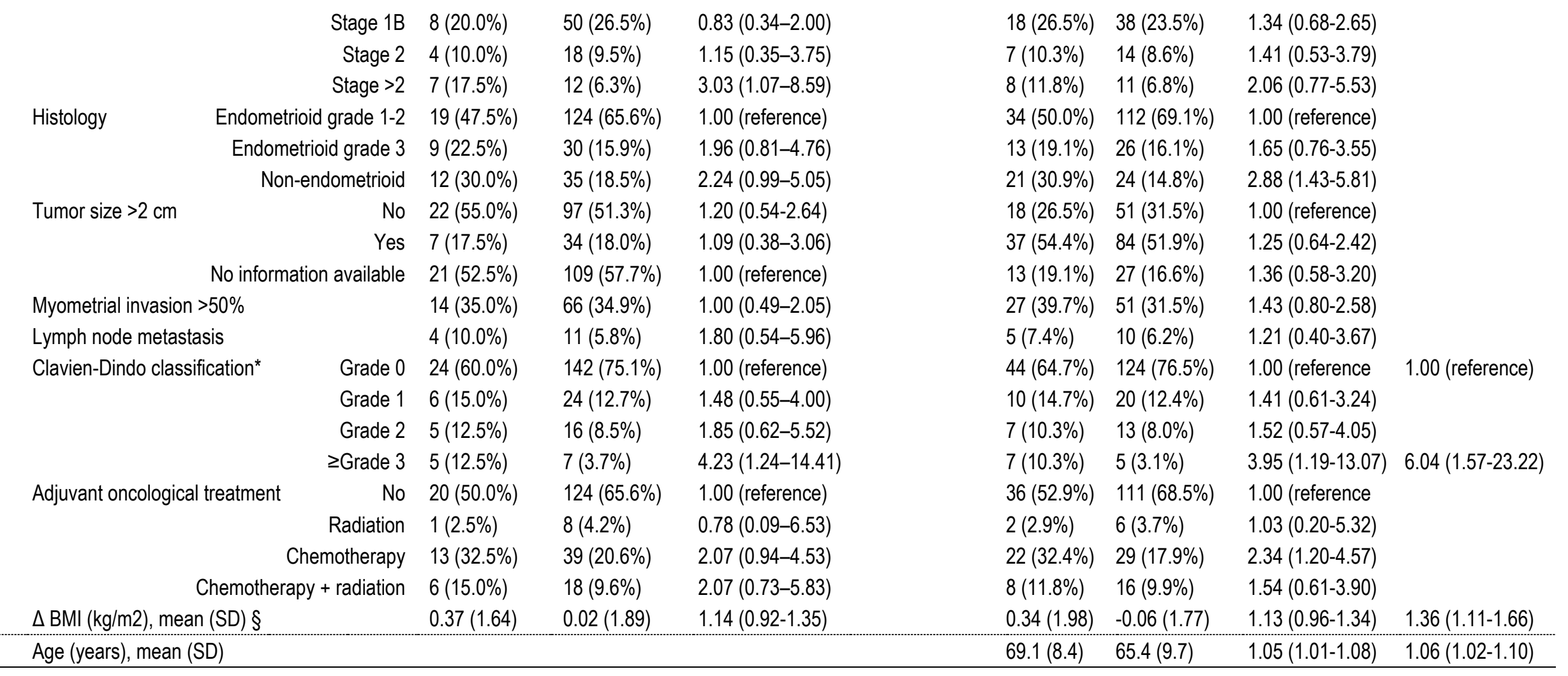

ASA - American Society of Anesthesiologist. BMI - body mass index. CI - confidence interval. EBV - estimated bleeding volume. LLL lower limb lymphedema. NSAID - non-steroidal anti-inflammatory drugs. OR -odds ratio. SD - standard deviation

* Clavien-Dindo classification of postoperative complications within 30 days.

$\S \Delta \mathrm{BMI}=\mathrm{BMI}$ at one year $-\mathrm{BMI}$ at baseline.

\# The preoperative data were analyzed separately in the first step. In addition to all factors and covariates depicted in the section intra- and postoperative data, the independent risk factors denoted under the section preoperative data were included in the multivariable analysis of intraand postoperative risk factors. Only aOR's of significant independent risk factors are denoted. 
Table 4. Risk factors for lymphedema related to lymphadenectomy. Lymphedema determined by clinical grading evaluated by lymphedema therapist and as patient-perceived swelling of the legs.

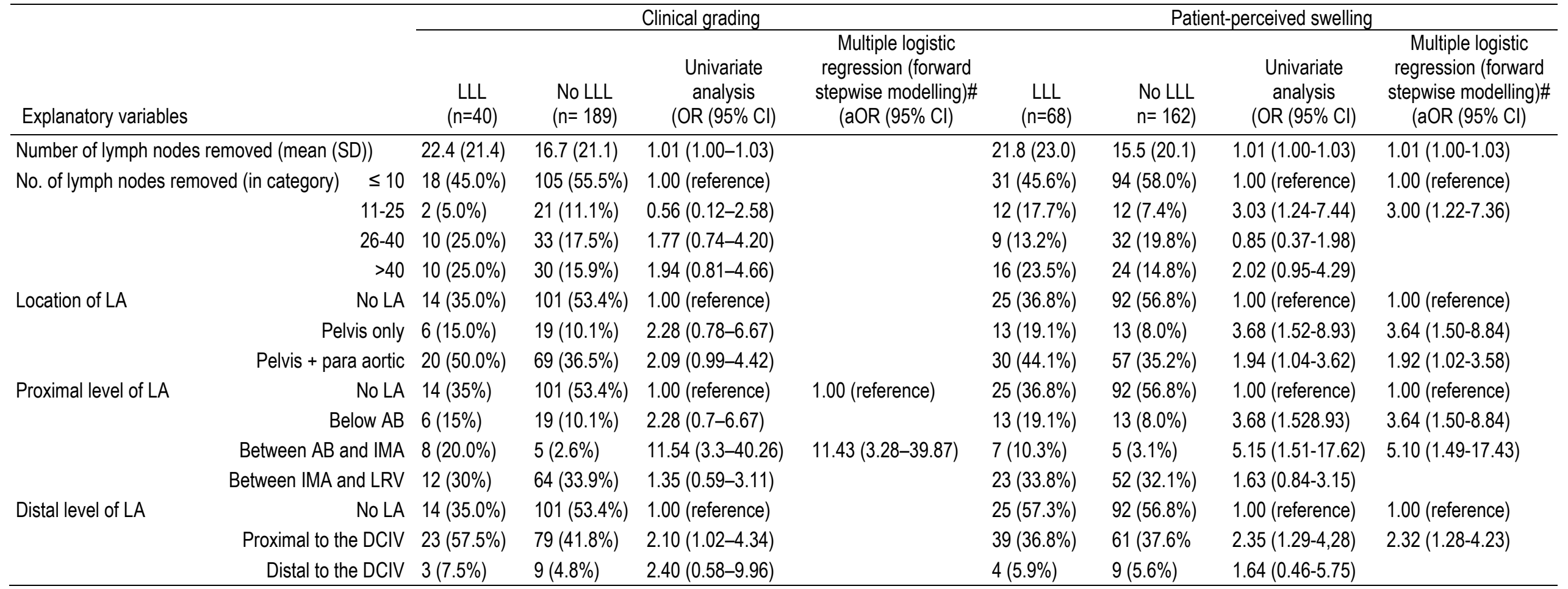

a - adjusted. AB - aortic bifurcation. CI - confidence interval. DCIV - deep circumflex iliac vein. LA - lymphadenectomy. LLL - lower limb lymphedema. LRV - left renal vein. IMA- inferior mesenteric artery. OR -odds ratio.

\# The multiple logistic regression forward stepwise models included adjustment for the independent risk factors depicted under intra- and postoperative data in Table 3 except the factor lymphadenectomy. Besides, potential factors that could influence lymphadenectomy (BMI, estimated bleeding volume, ASA classification, surgical method and mode of hysterectomy) were added simultaneously.

The explanatory variables depicted in Table 4 were analyzed individually in the multivariable models since these variables may be intercorrelated. Only aORs of significant independent risk factors are denoted. 
Table 5. Agreement between various methods of measuring lower limb lymphedema.

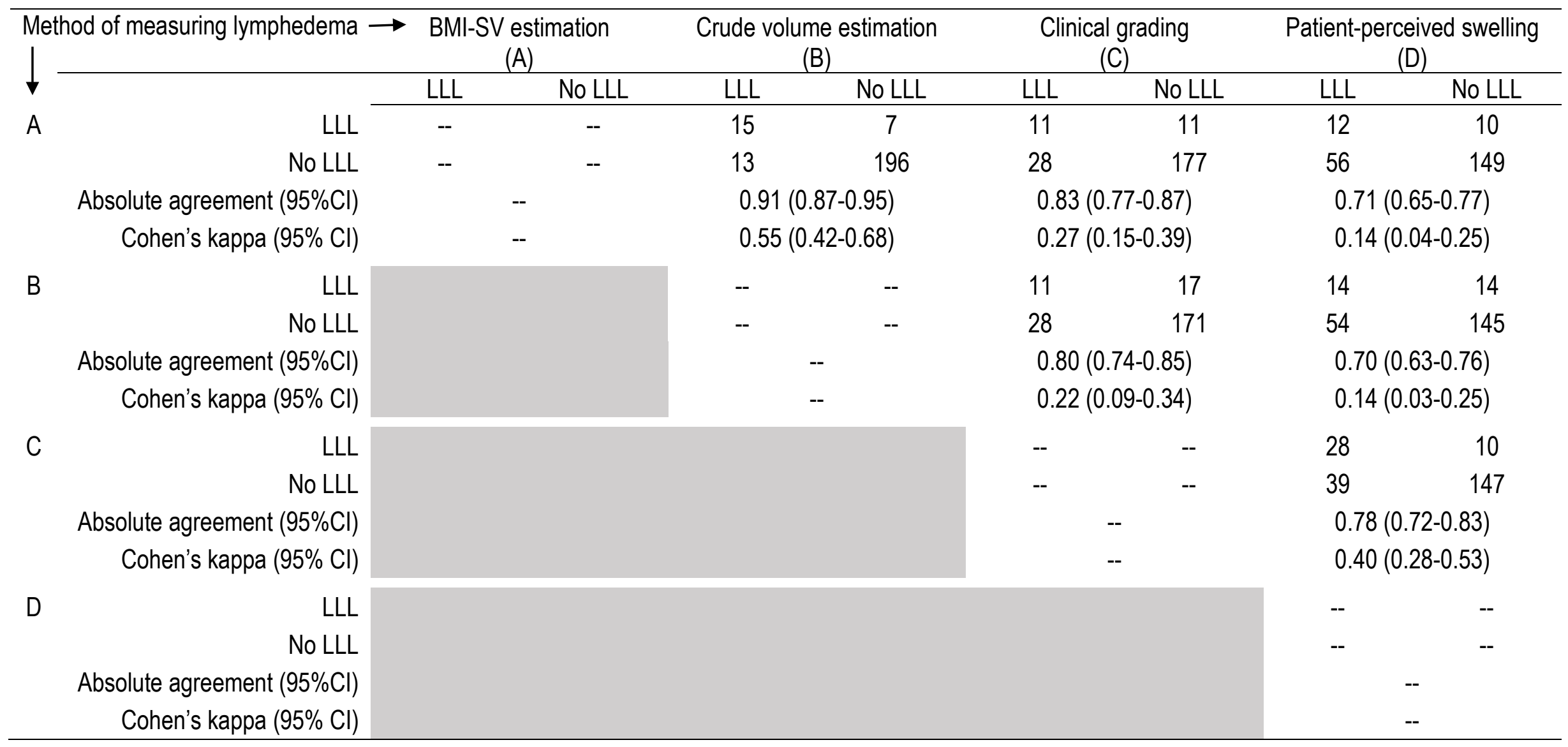

Figures denote number of women. BMI-SV - body mass index-standardized volume. CI - confidence interval. LLL - lower limb lymphedema 
47 Table 1. Risk factors for lower limb lymphedema determined as a volume increase $\geq 10 \%$ based on body mass index-standardized leg volume or crude leg volume.

49 Table 2. Risk factors for lymphedema related to lymphadenectomy. Lymphedema determined 50 as a volume increase $\geq 10 \%$ based on body mass index-standardized leg volume or crude leg

51 volume.

52 Table 3. Risk factors for lower limb lymphedema determined by clinical grading or as patient's perception of increased swelling of the legs.

54 Table 4. Risk factors for lymphedema related to lymphadenectomy. Lymphedema determined 55 by clinical grading evaluated by lymphedema therapist and as patient-perceived swelling of 56 the legs.

57 Table 5. Agreement between various methods of measuring lower limb lymphedema. 
60

61

62

63

64

65

We express our deep gratitude to all women who participated in the trial and to the all the coworkers. Without the participants and dedicated co-workers this study would not have been possible in our aim to bring knowledge to all the women that suffers from lymphedema.

The LASEC trial group consisted of (in alphabetic order of the hospitals):

Blekinge Hospital, Karlskrona: Eva Rundqvist, MD, Sarah Karlsson, RNM

Eksjö Highland Hospital: Lisbeth Liest, MD, Liselotte Roos, RN, Karin Norin Andersson, RPT

Falun Hospital: Åsa Nyberg, MD, PhD, Karin Ervard, RN, Helena Johansson, RPT

Gävle Hospital: Peter Smith, MD, PhD, Anette Henriksson, RN, Lena Larsson, Ulrika Ehn, RPT

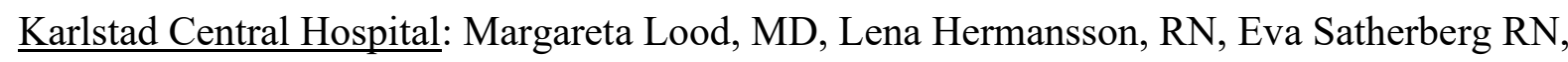
Eva Eliasson RPT

NÄL Hospital, Trollhättan: Eva Blank, MD, PhD, Inga-Lill Martinsson, RN, Marie de-France Westerling, RPT, Eva Mattsson RPT

Sahlgrenska University Hospital, Gothenburg: Åsa Åkesson, MD, Eva Rosén, RN, Birgitta Bååthe, RPT, Elisabeth Brodin, RPT, Matilda Möller, RPT

Skaraborg Hospital, Skövde: Lars Hogström, MD, Maarten Buimer, MD, PhD, Agneta Johansson, RN, Eva Andersson RN, Johanna Tallbacka, RPT, Sofie Eriksson, RPT

Sundsvall Regional Hospital: Lotta Andrèen, MD, PhD, Christine Näslund, RN, Ylva Vidgren, RPT 
University Hospital, Linköping: Preben Kjølhede, MD, PhD, Madelene Wedin, MD, Per Rosenberg, MD, PhD, Gabriel Lindahl, MD, PhD, Ninnie Borendal Wodlin, MD, PhD, Linda

Shosholli, RN, Åsa Rydmark Kersley, RN, MScN, Eva Ahlner, RPT

University Hospital of Umeå: Ulrika Ottander, MD, PhD, Jenny Eklund, RN, Åsa Sandström, Annika Falk, RPT

Uppsala University Hospital: Karin Glimskär Stålberg, MD, PhD, Charlotte Eklind, RN, Petra Strandh, RPT, Nicola Ingvast, RPT

Varberg Hospital: Janusz Marcickiewicz, MD, PhD, Pia Andersson, RN Lotti Källman, RN, Viveka Grant-Pedersen, RPT

Västervik Hospital: Anders Rosenmüller, MD, Carina Bergsten, RN, Camilla Dahlberg, RPT

Västmanland Hospital, Västerås: Lars Henning, MD, Andres Hess Engström, RPT

\section{Funding details}

The work was supported by The Swedish Cancer Society (Cancerfonden) (grant number CAN2013/620; recipient: PK), The Medical Research Council of Southeast Sweden (grant numbers FORSS-308611, FORSS-391311, FORSS-662141 and FORSS-858611; recipient: PK), Uppsala-Örebro Regional Research Council (grant number LUL-349271; recipient: KS) and unrestricted grants from the Scientific Council of the Region Halland (recipient: JM), the County Council of Östergötland (recipient: PK), and Linköping University (recipient: PK). 